\title{
A NEW PRESCRIPTION FOR THE MASS-LOSS RATES OF WC AND WO STARS
}

\author{
F. TRAMPER ${ }^{1,4}$, H. SANA ${ }^{2}$, AND A. DE KOTER ${ }^{2,3}$ \\ ${ }^{1}$ European Space Astronomy Centre (ESA/ESAC), Operations Department, Villanueva de la Cañada (Madrid), Spain; ftramper@sciops.esa.int \\ 2 Institute of Astrophysics, KU Leuven, Celestijnenlaan 200 D, B-3001, Leuven, Belgium \\ 3 Anton Pannekoek Institute for Astronomy, University of Amsterdam, P.O. Box 94249, 1090 GE Amsterdam, The Netherlands \\ Received 2016 February 10; revised 2016 October 12; accepted 2016 October 12; published 2016 December 13
}

\begin{abstract}
We present a new empirical prescription for the mass-loss rates of carbon- and oxygen-sequence Wolf-Rayet stars as a function of their luminosity, surface chemical composition, and initial metallicity. The new prescription is based on results of detailed spectral analyses of WC and WO stars and improves the often applied Nugis and Lamers relation. We find that the mass-loss rates of WC and WO stars (with $X=0$ and $Y \lesssim 0.98$ ) can be expressed as $\log \dot{M}=-9.20+0.85 \log \left(L / L_{\odot}\right)+0.44 \log Y+0.25 \log \left(Z_{\mathrm{Fe}} / Z_{\mathrm{Fe}, \odot}\right)$. This relation is based on mass-loss determinations that assume a volume-filling factor of 0.1 , but the prescription can easily be scaled to account for other volume-filling factors. The residual of the fit is $\sigma=0.06 \mathrm{dex}$. We investigated whether the relation can also describe the mass loss of hydrogen-free WN stars and showed that it can when an adjustment of the metallicity dependence $\left(\log \dot{M} \propto 1.3 \log \left(Z_{\mathrm{Fe}} / Z_{\mathrm{Fe}, \odot}\right)\right)$ is applied. Compared to that of Nugis and Lamers, $\dot{M}$ is less sensitive to the luminosity and the surface abundance, implying a stronger mass loss of massive stars in their late stages of evolution. The modest metallicity dependence implies that if WC or WO stars are formed in metal-deficient environments, their mass-loss rates are higher than currently anticipated. These effects may result in the formation of a larger number of SNe Ic and fewer black holes and may favor the production of superluminous SNe Ic through interaction with $\mathrm{C}$ - and $\mathrm{O}$-rich circumstellar material or dense stellar wind.
\end{abstract}

Key words: stars: evolution - stars: fundamental parameters - stars: mass-loss - stars: massive - stars: winds, outflows - stars: Wolf-Rayet

\section{INTRODUCTION}

Classical Wolf-Rayet (WR) stars are evolved massive stars characterized by dense, optically thick outflows, to which they owe their tell-tale emission-line spectra. Driven by radiation pressure (e.g., Gräfener \& Hamann 2005; Vink \& de Koter 2005), the winds of these stars require an efficient momentum transfer from the radiation to the gas, usually quantified by means of the wind performance number $\eta \equiv \dot{M} v_{\infty} /(L / c)$ (with $\dot{M}$ as the mass-loss rate, $v_{\infty}$ the terminal velocity of the wind, and $L$ the stellar luminosity). For WC and WO stars, $\eta$ reaches values of around 10 (e.g., Sander et al. 2012; Tramper et al. 2015), and most WN stars have $\eta$ close to unity (e.g., Hamann et al. 2006; Hainich et al. 2014, 2015). This indicates that multiple photon scatterings are required to drive the wind.

A good empirical knowledge of the outflow properties during the WR phase is necessary to understand the mechanism that drives the winds as well as to provide accurate values to be used in evolutionary models. The mass-loss efficiency in the WR phase strongly impacts the immediate pre-supernova evolution and determines the predicted type of supernova as well as the type of compact object that is produced.

Nugis \& Lamers (2000; hereafter, NL00) provide an empirical mass-loss prescription for WR stars as a function of luminosity and surface chemical composition. The NL00 rates are currently implemented in most evolutionary models, using either the separate $\mathrm{WN}$ and $\mathrm{WC}$ prescriptions (Equations (20) and (21) in NL00, e.g., in the Geneva models; Ekström et al. 2012) or the combined WR prescription (Equation (22) in NL00, e.g., in MESA; Paxton et al. 2011). However, the mass-loss rates of oxygen-sequence WR stars,

\footnotetext{
${ }^{4}$ ESA Research Fellow.
}

which have a very low surface helium abundance, cannot be reproduced by the NL00 prescriptions (Tramper et al. 2015).

In this paper, we provide a new prescription for the massloss rates of hydrogen-free WR stars, significantly improving predictions of the mass-loss rates during the WC and WO phases. The next section presents the calibration of this new prescription. The dependence of this prescription on stellar parameters and the parameter domain in which it is valid is then discussed in Section 3. We summarize our findings in Section 4.

\section{CALIBRATION OF WC AND WO STAR MASS LOSS}

The aim of this work is to revisit the prescription of WR mass-loss rates and to obtain a new calibration that is valid over the full parameter space covered by the WC and WO stars. To do this, we assume that the mass-loss rates $\left(\dot{M}\right.$, in $\left.M_{\odot} \mathrm{yr}^{-1}\right)$ of these stars can be described by a relation of the form

$$
\log \dot{M}=A+B \log \frac{L}{L_{\odot}}+C \log Y+D \log \frac{Z_{\mathrm{Fe}}}{Z_{\mathrm{Fe}, \odot}} .
$$

This relation is similar to that of NL00, i.e., the mass loss depends on the stellar luminosity $(L)$ and the surface helium mass fraction $(Y)$. In the case of hydrogen-free WR stars, the surface mass fraction of heavier elements is by definition $Z=1-Y$, and we do not include a separate term for this component. ${ }^{5}$ In WC and WO stars, $Z$ is effectively the sum of the high carbon and oxygen abundances, and the contribution from iron-like elements to this mass fraction is small. However, iron-group elements are expected to be the dominant wind

\footnotetext{
5 Inclusion of a $\log Z$ term does not yield a significantly better fit to the data, as verified by an $F$-test $(p(F)>0.8)$. The $\log Z$ coefficient is not significantly different from 0 . Such a fit further results in larger formal uncertainties in the derived parameters as a result of the strong correlation between $Y$ and $Z$.
} 
Table 1

Spectral Types and Parameters of The Calibration Stars

\begin{tabular}{|c|c|c|c|c|c|c|c|c|}
\hline$\#^{\mathrm{a}}$ & ID & Alt. ID & $\mathrm{SpT}$ & $\log L / L_{\odot}$ & $\log \dot{M}$ & $Y$ & $Z_{\mathrm{Fe}} / Z_{\mathrm{Fe}, \odot}$ & Reference \\
\hline 1 & $\mathrm{WR} 11^{\mathrm{b}}$ & $\gamma$ Vel & WC8 & 5.0 & -5.1 & 0.64 & 1.0 & De Marco et al. (2000) \\
\hline 2 & WR26 & & WN7/WCE & 6.1 & -4.01 & 0.80 & 1.0 & Sander et al. (2012) \\
\hline 3 & WR58 & & WN4/WCE & 5.15 & -4.80 & 0.975 & 1.0 & Sander et al. (2012) \\
\hline 4 & WR90 & HD 156385 & WC7 & 5.5 & -4.6 & 0.53 & 1.0 & Dessart et al. (2000) \\
\hline 5 & WR93b & & WO3 & 5.30 & -5.00 & 0.29 & 1.0 & Tramper et al. (2015) \\
\hline 6 & WR102 & & WO2 & 5.45 & -4.92 & 0.14 & 1.0 & Tramper et al. (2015) \\
\hline 7 & WR103 & HD 164270 & WC9 & 4.9 & -5.0 & 0.61 & 1.0 & Crowther et al. (2006) \\
\hline 8 & WR111 & HD 165763 & WC5 & 5.3 & -4.8 & 0.38 & 1.0 & Hillier \& Miller (1999) \\
\hline 9 & WR135 & HD 192103 & WC8 & 5.2 & -4.9 & 0.66 & 1.0 & Dessart et al. (2000) \\
\hline 10 & WR142 & & WO2 & 5.39 & -4.94 & 0.26 & 1.0 & Tramper et al. (2015) \\
\hline 11 & WR145 & & WN7/WCE & 5.8 & -4.35 & 0.935 & 1.0 & Sander et al. (2012) \\
\hline 12 & WR146 ${ }^{\mathrm{b}}$ & & WC5 & 5.7 & -4.5 & 0.76 & 1.0 & Dessart et al. (2000) \\
\hline 13 & Brey 7 & HD 32125, BAT 9 & WC4 & 5.44 & -4.8 & 0.65 & 0.5 & Crowther et al. (2002) \\
\hline 14 & Brey 8 & HD 32257, BAT 8 & WC4 & 5.42 & -4.9 & 0.45 & 0.5 & Crowther et al. (2002) \\
\hline 15 & Brey 10 & HD 32402, BAT 11 & WC4 & 5.70 & -4.5 & 0.66 & 0.5 & Crowther et al. (2002) \\
\hline 16 & Brey 43 & HD 37026, BAT 52 & WC4 & 5.65 & -4.5 & 0.46 & 0.5 & Crowther et al. (2002) \\
\hline 17 & Brey 50 & HD 37680, BAT 61 & WC4 & 5.68 & -4.4 & 0.74 & 0.5 & Crowther et al. (2002) \\
\hline 18 & Brey 74 & HD 269888, BAT 90 & WC4 & 5.44 & -4.8 & 0.45 & 0.5 & Crowther et al. (2002) \\
\hline 19 & Brey 93 & BAT 123 & WO3 & 5.20 & -5.14 & 0.30 & 0.5 & Tramper et al. (2015) \\
\hline 20 & [L72]LH41-1042 & & WO4 & 5.26 & -5.05 & 0.22 & 0.5 & Tramper et al. (2015) \\
\hline 21 & DR1 in IC1613 & & WO3 & 5.68 & -4.76 & 0.44 & 0.15 & Tramper et al. (2013) \\
\hline
\end{tabular}

Notes.

${ }^{\text {a }}$ Used as labels in Figures 1 and 2.

${ }^{\mathrm{b}} \mathrm{WR}+\mathrm{O}$ binary. The contribution from the $\mathrm{O}$ star was taken into account in the spectroscopic analysis.

drivers even at high carbon and oxygen abundances (e.g., Crowther et al. 2002; Vink \& de Koter 2005). We therefore explicitly include this dependence on the iron mass fraction $Z_{\mathrm{Fe}}$ in Equation (1). This ensures that the derived prescription is valid for stars in different metallicity environments. We adopt the solar abundances from Asplund et al. (2009; i.e., $\left.Z_{\odot}=0.014\right)$.

We use the parameters of the WC and WO stars listed in Table 1 to derive the coefficients of Equation (1). These stars have been selected using the following criteria: (1) the spectrum was quantitatively analyzed using non-LTE atmosphere models that include line blanketing and account for wind clumping, and (2) the surface abundances of carbon and oxygen have been modeled in the analysis (i.e., no grid-based analyses where the abundances were fixed). All WC and WO stars in the sample were analyzed using CMFGEN (Hillier \& Miller 1998), and the WN/WC stars were examined with the Potsdam WR (POWR) code (Gräfener et al. 2002; Hamann \& Gräfener 2004). The WN/WC stars are included to provide calibration points at high $Y$ values and to increase the sample size. Exclusion of these stars results in an essentially identical fit, but with larger error bars on the coefficients. Two of the Galactic WC stars (WR11 and WR146) are WR+O binaries. While this has been taken into account in the spectroscopic analysis, we note that their properties may be more uncertain.

All mass-loss rates in Table 1 correspond to a volume-filling factor of $f_{\mathrm{c}}=0.1$, which is typical for WR stars. The predicted mass-loss rates are thus only valid if the winds of the calibration stars indeed have this volume-filling factor, but can easily be scaled to account for other values of $f_{\mathrm{c}}$ (using $\dot{M} \propto f_{\mathrm{c}}^{0.5}$ ).

Although not all spectral subtypes are represented, the sample of 21 stars provides a good coverage of the relevant parameter space, i.e., spanning a range of luminosities $\left(4.9 \leqslant \log \left(L / L_{\odot}\right) \leqslant 6.1 ;\right.$ Figure 1$)$ and surface abundances
$(0.14 \leqslant Y \leqslant 0.98$; Figure 2$)$. Twelve stars are located in the Milky Way (MW; $Z_{\mathrm{Fe}}=Z_{\mathrm{Fe}, \odot}$ ), and eight in the Large Magellanic Cloud (LMC; $\left.Z_{\mathrm{Fe}}=0.5 Z_{\mathrm{Fe}, \odot}\right)$. The WO star DR1 located in IC1613 provides a valuable third metallicity point at $Z_{\mathrm{Fe}}=0.15 Z_{\mathrm{Fe}, \odot}$.

The coefficients of Equation (1) were derived by a linear regression to the data of Table 1 . The resulting mass-loss prescription with $1 \sigma$ uncertainties on the coefficients is

$$
\begin{aligned}
\log \dot{M}= & -9.20( \pm 0.35)+0.85( \pm 0.06) \log \frac{L}{L_{\odot}} \\
& +0.44( \pm 0.08) \log Y+0.25( \pm 0.08) \log \frac{Z_{\mathrm{Fe}}}{Z_{\mathrm{Fe}, \odot}}
\end{aligned}
$$

The mass-loss rates that are predicted by the new prescription are compared to the calibration values in Figures 1 and 2. The figures also show the mass-loss rates predicted by the NL00 prescription (their Equation (22)), which is often applied in evolutionary models. The NLO0 rates have been scaled with the theoretically predicted metallicity dependence of $\dot{M} \propto Z_{\mathrm{Fe}}^{0.66}$ (Vink \& de Koter 2005).

The mass-loss rates from the new prescription match the calibration values better than those from the NL00 prescription. The most significant improvement is for the WO stars, where residuals decrease from $0.1-0.5 \mathrm{dex}$ for NLO0 to less than 0.05 dex for the new prescription. Over the whole calibration sample, the standard deviation of the mass-loss rates from the new prescription compared to the calibration values is $\sigma=0.06 \mathrm{dex}$, versus $\sigma=0.18 \mathrm{dex}$ for the NL00 rates ( $\sigma=0.17 \mathrm{dex}$ for the WC prescription of NL00; their Equation (21)). The mean deviation of the mass-loss rates of the new prescription from the calibration values is $10^{-4} \mathrm{dex}$, indicating that there is no systematic offset to higher or lower mass-loss rates. These numbers indicate that the new 


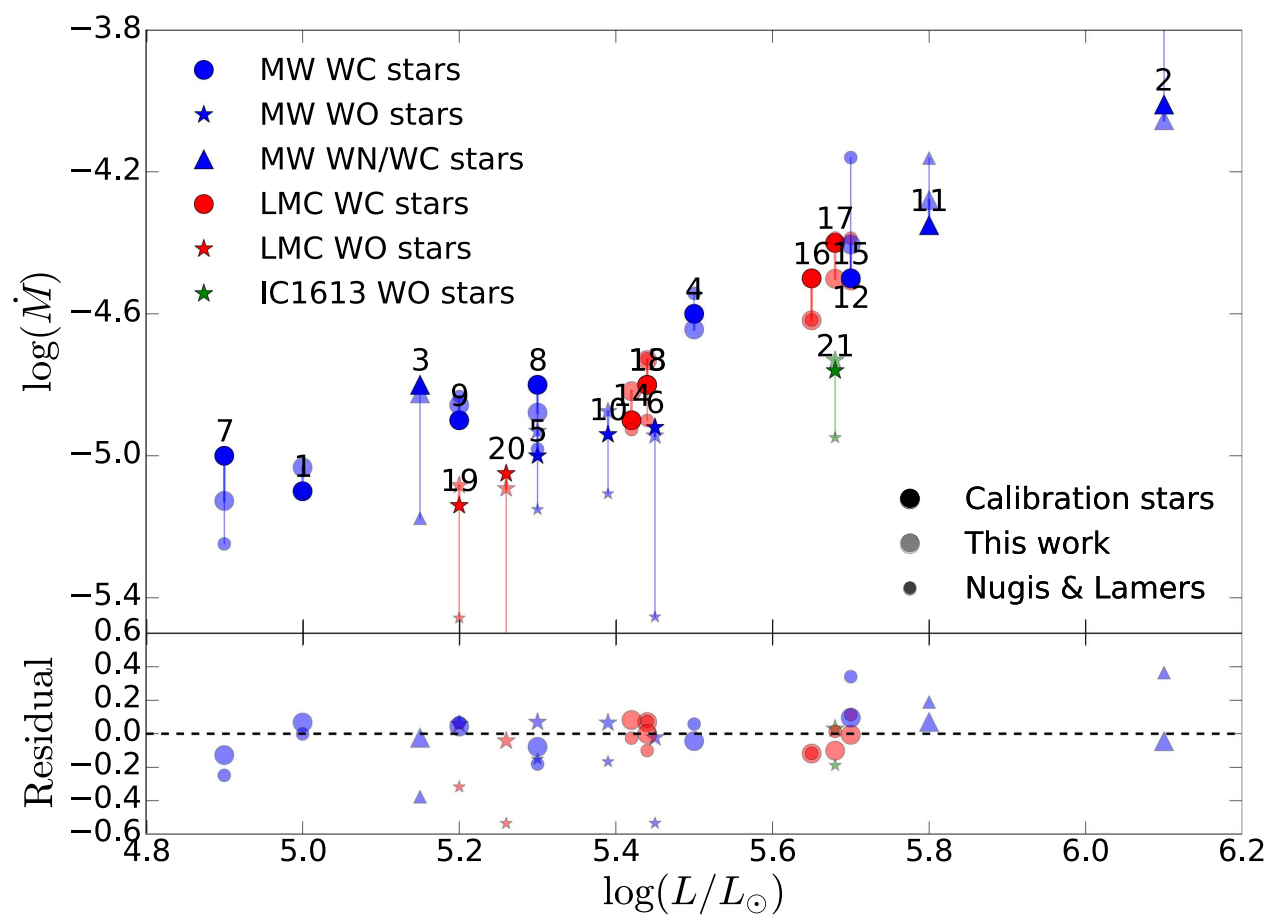

Figure 1. Measured mass-loss rates vs. luminosity of our sample stars (large solid symbols) compared to values computed by the prescription from this work (large transparent symbols) and NL00 (small transparent symbols). The bottom panel shows the residuals for both prescriptions.

prescription provides a significant improvement in the prediction of the mass-loss rates of WC and WO stars.

\section{DISCUSSION}

In this section, we first compare our results to the sample of single Galactic WC stars from Sander et al. (2012). Then we discuss the implications of the change in dependence of the mass-loss rates on luminosity, abundance, and metallicity for the late stages of evolution of massive stars.

\subsection{Galactic WC Stars}

Sander et al. (2012) analyzed all known presumed-single Galactic WC stars using a grid of models from the Potsdam WR (POWR) code. In the analysis, the surface mass fractions of helium, carbon, and oxygen were fixed to $0.55,0.45$, and 0.05 , respectively. This may affect the derived luminosities and mass-loss rates for stars whose abundances deviate significantly from these values. In Figure 3 we compare their results to the predictions from the new mass-loss prescription and those from the NL00 prescriptions.

The four WC stars that are both in the calibration sample and in the Sander et al. (2012) sample are marked in the plot. The considerable offset in luminosity and mass loss for WR90, WR103, and WR135 can be explained by the differences in the adopted distances $(0.8 \mathrm{kpc}$ versus $1.55 \mathrm{kpc}$ for WR90, $2.4 \mathrm{kpc}$ versus $1.9 \mathrm{kpc}$ for WR103, and $1.7 \mathrm{kpc}$ versus $1.4 \mathrm{kpc}$ for WR135). However, the trend of the mass-loss rate with luminosity is conserved for these stars (see also discussion below), all of which have helium abundances relatively close to $Y=0.55(Y=0.53$ for WR90, $Y=0.61$ for WR103, and $Y=0.66$ for WR135).

For the WC5 star WR111, the distances assumed in both analyses are comparable $(1.55 \mathrm{kpc}$ versus $1.6 \mathrm{kpc})$. Here, the offset between the derived luminosities and mass-loss rates may be a result of the assumed abundances in Sander et al. (2012), as the helium abundance derived by the detailed modeling is $Y=0.38$. If the other early-type WC stars have comparable abundances, this may explain the larger offset of these stars to the predictions from each of the prescriptions (see Figure 3; WC4 and WC5 subtypes marked with star symbols).

\subsection{Dependences and Implications}

The dependence of the mass-loss rates of hydrogen-free WR stars on luminosity derived in this work $\left(\dot{M} \propto L^{0.85 \pm 0.06}\right)$ is consistent with those derived for WC stars by NL00 $\left(\dot{M} \propto L^{0.84 \pm 0.17}\right.$; their Equation (21)) and Sander et al. (2012; $\dot{M} \propto L^{0.83 \pm 0.11}$ ). This dependence can naturally be explained by the results of Sander et al. (2012), who find that the transformed radius is proportional to the temperature squared. For fixed terminal wind velocities $\left(v_{\alpha}\right)$, this relation results in a $\dot{M} \propto L^{0.75}$ dependence. The slightly steeper dependence on $L$ is the result of an increasing $v_{\alpha}$ toward earlier spectral subtypes (see Sander et al. 2012 for a discussion).

The luminosity dependence of the combined $\mathrm{WN}+\mathrm{WC}$ prescription of NL00 is much steeper $\left(\dot{M} \propto L^{1.29}\right.$; their Equation (22)). This results in an underprediction of the mass-loss rate for low luminosities and an overprediction for high luminosities (see Figure 3). This effect reaches up to $0.2 \mathrm{dex}$ in $\dot{M}$ for the lowest/highest luminosities. Implemented in stellar evolution models, these modifications in mass-loss properties may impact the surface abundance ratio of carbon and oxygen, which is essential in constraining the elusive ${ }^{12} \mathrm{C}$ $(\alpha, \gamma)^{16} \mathrm{O}$ thermonuclear reaction rate (e.g., Grafener et al. 1998). The NL00 prescription for WN stars also has a much steeper luminosity dependence $\left(\dot{M} \propto L^{1.63}\right.$; their Equation (21)). However, the transformed-radius argument given above should hold for these stars, and this high value is likely to be a result of the very strong dependence on the 


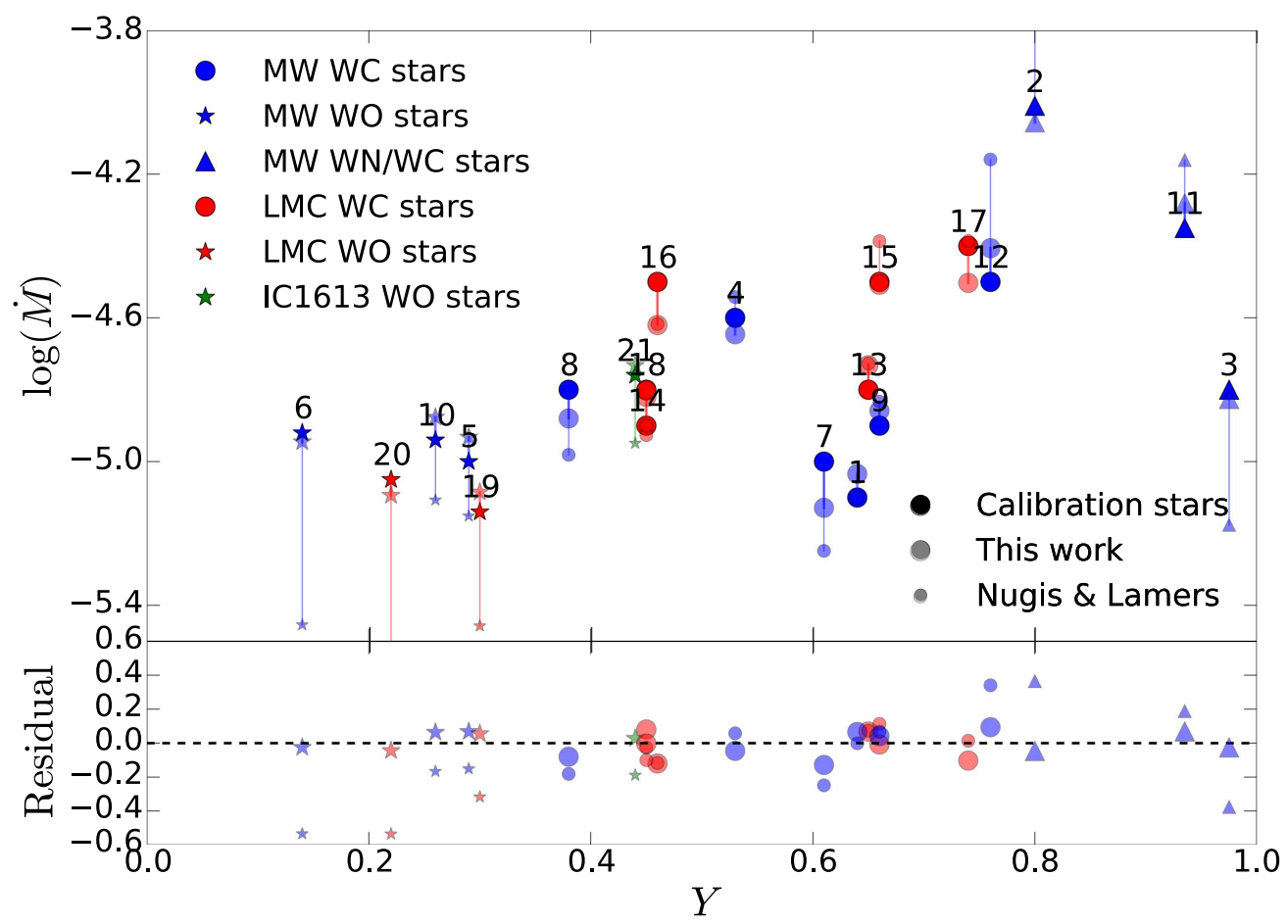

Figure 2. Same as Figure 1, but as a function of the surface helium mass fraction.

helium abundance $\left(\dot{M} \propto Y^{2.22}\right)$ in this prescription due to the inclusion of hydrogen-containing WN stars.

The largest impact on the late stages of evolution of massive stars comes from the dependence of the mass-loss rate on the surface abundances. The combined NL00 prescription gives $\dot{M} \propto Y^{1.29} Z^{0.5}$. The abundance dependence of the NL00 WC relation is even stronger: $\dot{M} \propto Y^{2.04} Z^{1.04}$. The strong $Y$ dependence implies that the mass loss decreases strongly toward the late stages of evolution, an effect that is not in agreement with the derived mass-loss rates of the WO stars. The weaker $\dot{M} \propto Y^{0.44}$ dependence derived in this work does produce mass-loss rates in agreement with those derived for both the WC and WO stars. This implies that the mass-loss rates of WC and WO stars are considerably higher toward the later stages of evolution, where the surface helium abundance becomes low (see Figure 2).

The combined effect of the luminosity and abundance dependences is that more mass than currently predicted is lost during the last $\sim 40 \%$ of the core helium-burning phase and the post-helium-burning phase. Implementation in evolutionary models is needed to assess the impact of this extra mass loss on the lifetimes of the WC and WO stars and on the properties of the direct progenitor stars of supernovae. Potential implications are that these properties favor an increased number of SNe Ic in either single star or close binary evolution. Our findings may also be relevant to the discussion on the nature of superluminous SNe Ic that appear to be associated with faint and metal-poor galaxies (Chomiuk et al. 2011; Neill et al. 2011; Quimby et al. 2011). Among the scenarios proposed to explain these events (see, e.g., Inserra et al. 2013 for a discussion) is the interaction of the supernova ejecta with a massive carbon- and oxygen-rich circumstellar medium (Blinnikov \& Sorokina 2010) or with the dense wind of the progenitor (Chevalier \& Irwin 2011; Ginzburg \& Balberg 2012). Furthermore, a higher mass loss in the hydrogen-free WR phase would lead to a decrease in the number of black holes produced.
The metallicity dependence of the mass-loss prescription $\left(\dot{M} \propto Z_{\mathrm{Fe}}^{0.25}\right)$ is also weaker than that predicted by theory for WC stars $\left(\dot{M} \propto Z_{\mathrm{Fe}}^{0.66}\right.$; Vink \& de Koter 2005) as well as that empirically derived from Galactic and LMC WC stars alone $\left(\dot{M} \propto Z_{\mathrm{Fe}}^{\sim 0.5}\right.$; Crowther et al. 2002). To verify that the derived metallicity dependence is not dominated by the single calibration point at $0.15 Z_{\odot}$, we repeated the fit excluding this data point. This results in nearly identical values of coefficients $A, B$, and $C$ of Equation (1) and their errors (small changes at the third decimal). The derived value of the metallicity dependence is $D=0.20 \pm 0.12$, in good agreement with the value derived in Equation (2).

This weak dependence gives rise to stronger winds for WC and WO stars in low-metallicity environments than currently predicted, in agreement with the high mass-loss rate of the WO star in IC1613. However, the exposure of the deep layers to helium-burning products, necessary to produce WC and WO stars, depends on the mass-loss history in previous evolutionary stages, where the metallicity dependence of the stellar winds is found to be much higher (Equation (3)). Thus, it is harder for WC- and WO-type stars to form in low-metallicity environments, which may not be possible by mass loss through stellar winds alone. Instead, alternative mass-loss mechanisms, such as eruptions or mass transfer to a companion star, may be needed. The fact that no WC stars and only two WO stars are known at metallicities below that of the LMC suggests that their formation at low metallicities requires a very specific evolutionary history. However, if these stars do form, we find that their winds are relatively strong for their metallicity.

\subsection{Hydrogen-free WN Stars}

To assess in which domain of parameter space the new massloss prescription remains valid, we evaluate the predicted massloss rates of hydrogen-free WN stars (with $X=0$ and $Y \gtrsim 0.98)$ at various metallicities. 


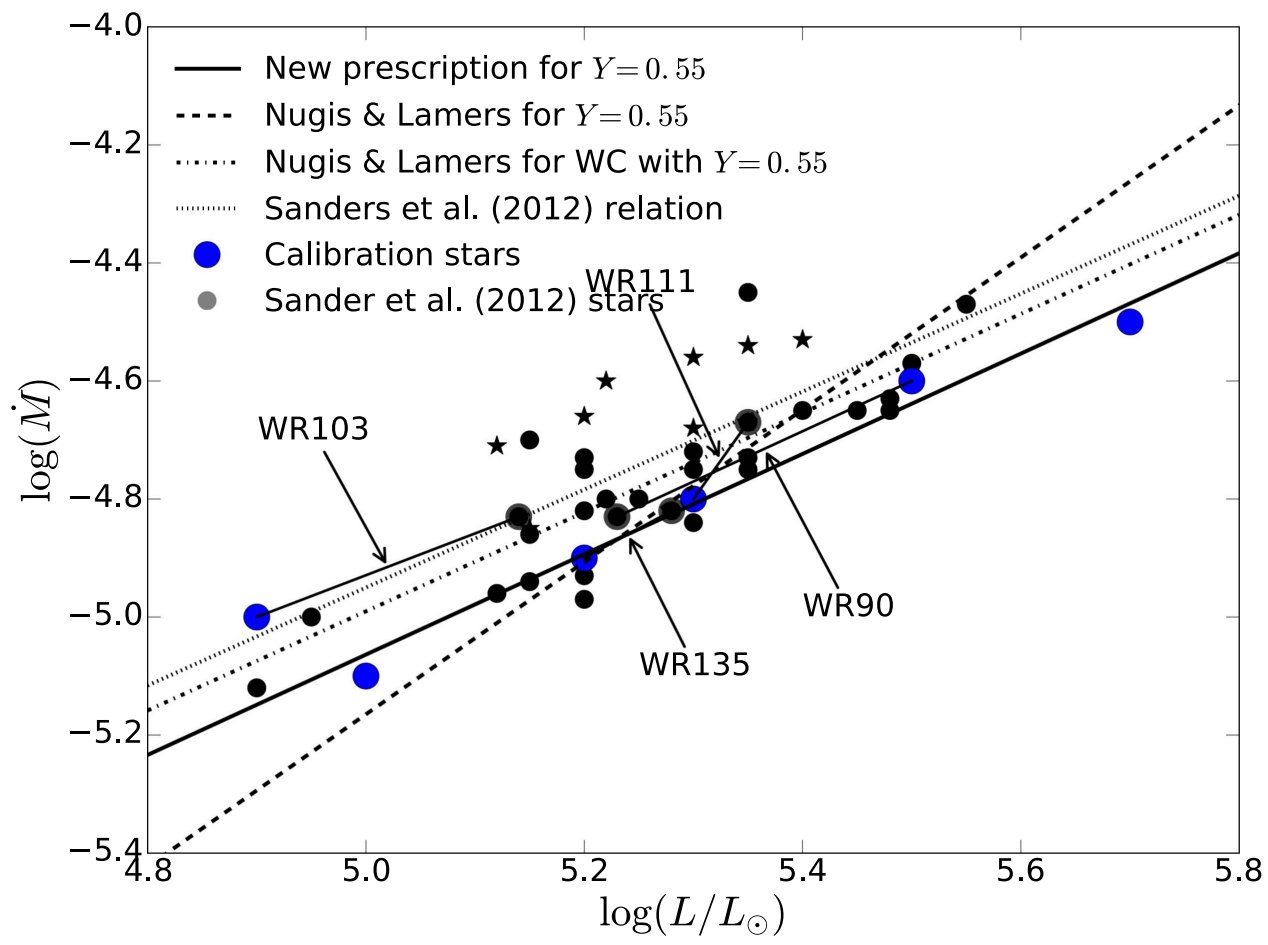

Figure 3. Comparison of mass-loss rates from the new prescription and the NL00 prescriptions to those of the Galactic WC stars analyzed by Sander et al. (2012). The mass loss vs. luminosity relation obtained by Sander et al. (2012) is also indicated. The four WC stars that are in our calibration sample are indicated by the larger symbols, with the values from Sander et al. (2012) in black and the values used in this work in blue. The two calibration WC stars that are not in the Sander et al. (2012) sample are also shown in blue symbols. Early-type WC stars (WC4 and WC5) are indicated by star symbols.

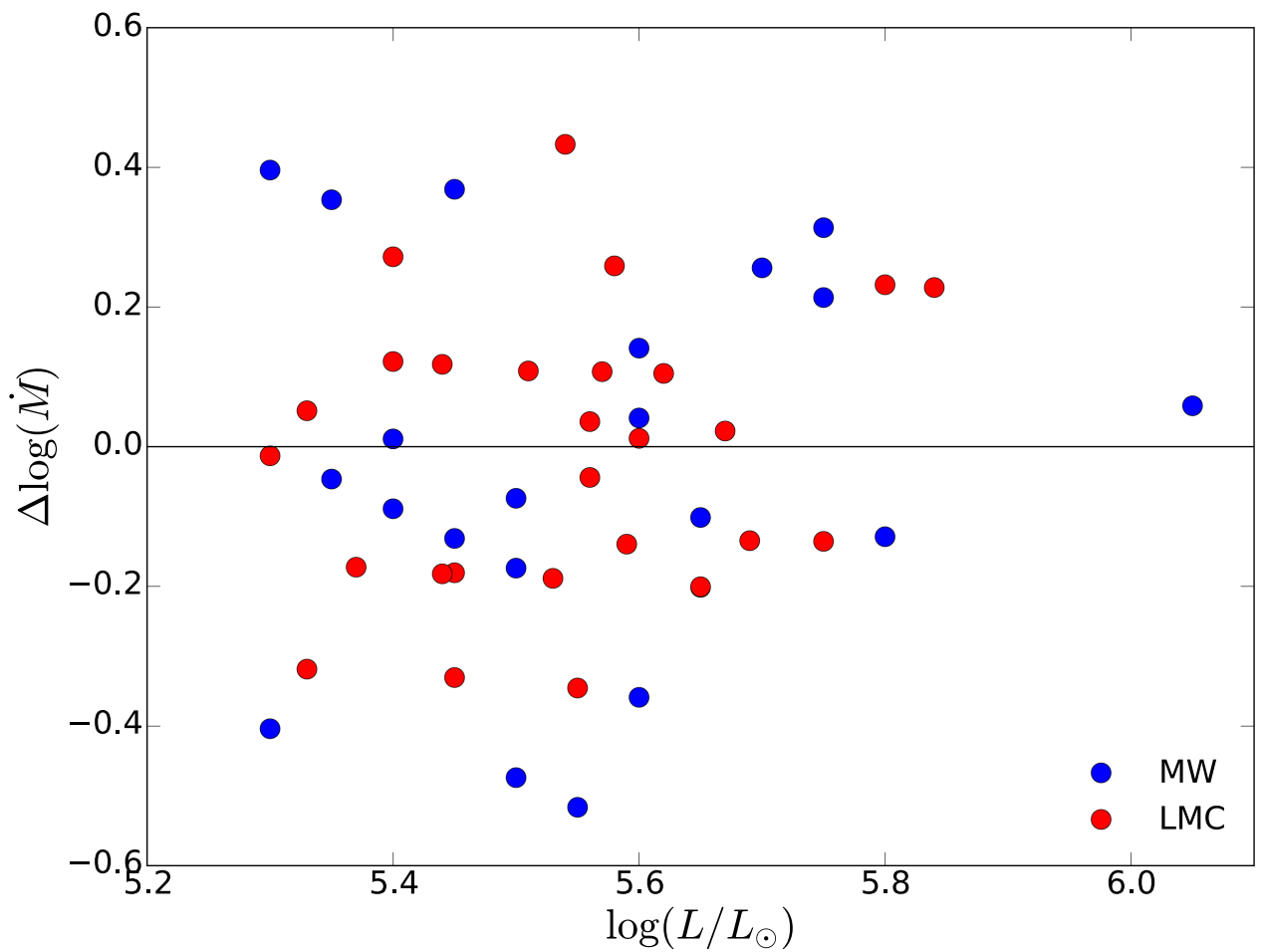

Figure 4. Residuals of the mass-loss rates $\left(\Delta \log \dot{M}=\log \dot{M}_{\text {observed }}-\log \dot{M}_{\text {predicted }}\right)$ computed for hydrogen-free WN stars, taking into account the stronger metallicity dependence of Equation (3). Stellar parameters from Hamann et al. (2006; MW) and Hainich et al. (2014; LMC).

For the hydrogen-free, presumed-single Galactic WN stars analyzed by Hamann et al. (2006), our prescription provides mass-loss rates with an accuracy comparable to those of the NL00 prescription, i.e., with a standard deviation of $0.2 \mathrm{dex}$ (see Figure 4). For the presumed-single hydrogen-free WN stars in the LMC studied by Hainich et al. (2014), the mass-loss rates are significantly overpredicted, suggesting that the dependence on initial metallicity of Equation (2) does not hold for WN stars. This is in line with the results from Hainich et al. (2015), who derive an empirical metallicity dependence for all 
WN stars (including those with hydrogen) using Galactic, M31, LMC, and Small Magellanic Cloud (SMC) WN stars. They find $\dot{M} \propto Z_{\mathrm{Fe}}^{1.4}$-thus, a much stronger dependence on initial metallicity.

To assess if Equation (2) would be valid with a different dependence on initial metallicity, we fit Equation (1) to the results for hydrogen-free WN stars in the Milky Way and LMC from Hamann et al. (2006) and Hainich et al. (2014), keeping $A, B$, and $C$ fixed to the values of Equation (2). This approach is motivated by the good results for the Galactic hydrogen-free WN stars, where the dependence on initial metallicity drops. This indicates that the luminosity dependence holds, as, for hydrogen-free WN stars, $Y$ does not vary between stars with the same initial metallicity (e.g., Hamann et al. 2006). We find a metallicity dependence of $\dot{M} \propto Z_{\mathrm{Fe}}^{1.3 \pm 0.2}$, in excellent agreement with the results from Hainich et al. (2015). Thus, the mass-loss rates of hydrogen-free WN stars can be described as

$$
\begin{aligned}
\log \dot{M}= & -9.20( \pm 0.35)+0.85( \pm 0.06) \log \frac{L}{L_{\odot}} \\
& +0.44( \pm 0.08) \log Y+1.3( \pm 0.2) \log \frac{Z_{\mathrm{Fe}}}{Z_{\mathrm{Fe}, \odot}} .
\end{aligned}
$$

With this dependence on metallicity, the prescription reproduces the mass-loss rates of the hydrogen-free LMC WN stars with a standard deviation of 0.2 dex and no systematic offset, i.e., with an accuracy comparable to that of the Galactic case (see Figure 4).

A change in metallicity dependence between $\mathrm{WN}$ and $\mathrm{WC}$ stars is predicted by Vink \& de Koter (2005), who find that in the metallicity range discussed here, the exponent of the $Z_{\mathrm{Fe}}$ dependence decreases from 0.86 for hydrogen-poor WN stars to 0.66 for WC stars. However, while the trend is in the right direction, the amplitude of the change in the exponent that reproduces the observations is about a factor of five larger than theory predicts. Combined with the shallow dependence on $Y$, this likely indicates that as the carbon abundance increases, this element becomes a more important driver of the wind.

We also compared our findings to WN stars that do contain hydrogen in the Galaxy, LMC, and SMC. For the sample of seven SMC WN stars studied by Hainich et al. (2015), spanning $X=0.2-0.5$, our prescription matches the observed mass-loss rates with an accuracy comparable to that of the hydrogen-free WN stars in the Galaxy and LMC. However, for the WN stars with hydrogen in the Galaxy and LMC, the results are poor-and more so for sources with higher $X$.

We conclude that our mass-loss prescription behaves well for hydrogen-free $\mathrm{WN}$ stars, provided that the steeper metallicity dependence of $\dot{M} \propto Z_{\mathrm{Fe}}^{1.3}$ is taken into account. For WN stars that have a significant surface hydrogen mass fraction, our prescription is less accurate.

\section{SUMMARY}

We have presented a new prescription for the mass-loss rates of hydrogen-free WR stars as a function of their luminosity, surface abundances, and metallicity. The prescription (Equations (2) and (3)) is based on the derived mass-loss rates of WC and WO stars in the Milky Way, LMC, and IC1613 galaxies. Equation (2) is valid for hydrogen-free WR stars with surface helium mass fractions $Y \lesssim 0.98$ with a precision of $\sigma=0.06$ dex. Equation (3) is valid for hydrogen-free WR stars with helium mass fractions $Y \gtrsim 0.98$, albeit with larger residuals ( $\sigma=0.2$ dex, comparable to that of the NL00 prescriptions). In practice this means that the prescription is valid for all $\mathrm{WC}$ and $\mathrm{WO}$ stars and for the hydrogen-free $(X=0)$ subset of WN stars. Future implementation in evolutionary codes will allow us to quantify the impact on the duration of late evolutionary stages and on the nature and properties of the final supernova explosion and the compact remnant.

We are grateful to Dr. Ehsan Moravveji for his insightful discussions.

\section{REFERENCES}

Asplund, M., Grevesse, N., Sauval, A. J., \& Scott, P. 2009, ARA\&A, 47, 481 Blinnikov, S. I., \& Sorokina, E. I. 2010, arXiv:1009.4353

Chevalier, R. A., \& Irwin, C. M. 2011, ApJL, 729, L6

Chomiuk, L., Chornock, R., Soderberg, A. M., et al. 2011, ApJ, 743, 114

Crowther, P. A., Dessart, L., Hillier, D. J., Abbott, J. B., \& Fullerton, A. W. 2002, A\&A, 392, 653

Crowther, P. A., Morris, P. W., \& Smith, J. D. 2006, ApJ, 636, 1033

De Marco, O., Schmutz, W., Crowther, P. A., et al. 2000, A\&A, 358, 187

Dessart, L., Crowther, P. A., Hillier, D. J., et al. 2000, MNRAS, 315, 407

Ekström, S., Georgy, C., Eggenberger, P., et al. 2012, A\&A, 537, A146

Ginzburg, S., \& Balberg, S. 2012, ApJ, 757, 178

Gräfener, G., \& Hamann, W.-R. 2005, A\&A, 432, 633

Grafener, G., Hamann, W.-R., Hillier, D. J., \& Koesterke, L. 1998, A\&A, 329,190

Gräfener, G., Koesterke, L., \& Hamann, W.-R. 2002, A\&A, 387, 244

Hainich, R., Pasemann, D., Todt, H., et al. 2015, A\&A, 581, A21

Hainich, R., Rühling, U., Todt, H., et al. 2014, A\&A, 565, A27

Hamann, W.-R., \& Gräfener, G. 2004, A\&A, 427, 697

Hamann, W.-R., Gräfener, G., \& Liermann, A. 2006, A\&A, 457, 1015

Hillier, D. J., \& Miller, D. L. 1998, ApJ, 496, 407

Hillier, D. J., \& Miller, D. L. 1999, ApJ, 519, 354

Inserra, C., Smartt, S. J., Jerkstrand, A., et al. 2013, ApJ, 770, 128

Neill, J. D., Sullivan, M., Gal-Yam, A., et al. 2011, ApJ, 727, 15

Nugis, T., \& Lamers, H. J. G. L. M. 2000, A\&A, 360, 227

Paxton, B., Bildsten, L., Dotter, A., et al. 2011, ApJS, 192, 3

Quimby, R. M., Kulkarni, S. R., Kasliwal, M. M., et al. 2011, Natur, 474, 487

Sander, A., Hamann, W.-R., \& Todt, H. 2012, A\&A, 540, A144

Tramper, F., Gräfener, G., Hartoog, O. E., et al. 2013, A\&A, 559, A72

Tramper, F., Straal, S. M., Sanyal, D., et al. 2015, A\&A, 581, A110

Vink, J. S., \& de Koter, A. 2005, A\&A, 442, 587 